\title{
Nebulised amphotericin B to eradicate Candida colonisation from the respiratory tract in critically ill patients receiving selective digestive decontamination: a cohort study
}

David SY Ong ${ }^{1,2,3^{*}}$, Peter MC Klein Klouwenberg ${ }^{1,2,3}$, Cristian Spitoni ${ }^{2,4}$, Marc JM Bonten ${ }^{2,3}$ and Olaf L Cremer ${ }^{1}$

\begin{abstract}
Introduction: Colonisation of the lower respiratory tract with Candida species occurs in 25\% of mechanically ventilated critically ill patients, and is associated with increased morbidity. Nebulised amphotericin B has been used to eradicate Candida as part of selective decontamination of the digestive tract (SDD) protocols, but its effectiveness is unknown. We aimed to determine the effectiveness of nebulised amphotericin B in eradicating Candida respiratory tract colonisation in patients receiving SDD.

Methods: We included consecutive mechanically ventilated patients during a four-year period. Microbiological screening was performed upon admission and twice weekly thereafter according to a standardised protocol. A colonisation episode was defined as the presence of Candida species in two consecutive sputum samples taken at least one day apart. To correct for time-varying bias and possible confounding, we used a multistate approach and performed time-varying Cox regression with adjustment for age, disease severity, Candida load at baseline and concurrent corticosteroid use.
\end{abstract}

Results: Among 1,819 patients, colonisation with Candida occurred 401 times in 363 patients; 333 of these events were included for analysis. Decolonisation occurred in 51 of 59 episodes (86\%) and in 170 of 274 episodes (62\%) in patients receiving and not receiving nebulised amphotericin $B$, respectively. Nebulised amphotericin $B$ was associated with an increased rate of Candida eradication (crude HR 2.0; 95\% Cl 1.4 to 2.7, adjusted HR 2.2; $95 \% \mathrm{Cl}$ 1.6 to 3.0). Median times to decolonisation were six and nine days, respectively. The incidence rate of ventilatorassociated pneumonia, length of stay and mortality did not differ between both groups.

Conclusions: Nebulised amphotericin B reduces the duration of Candida colonisation in the lower respiratory tracts of mechanically ventilated critically ill patients receiving SDD, but data remain lacking that this is associated with a meaningful improvement in clinical outcomes. Until more evidence becomes available, nebulised amphotericin B should not be used routinely as part of the SDD protocol.

\footnotetext{
*Correspondence: d.s.y.ong@umcutrecht.nl

'Department of Intensive Care Medicine, University Medical Center Utrecht,

Heidelberglaan 100, 3584, CX Utrecht, The Netherlands

${ }^{2}$ Department of Medical Microbiology, University Medical Center Utrecht,

Heidelberglaan 100, 3584, CX Utrecht, The Netherlands

Full list of author information is available at the end of the article
} 


\section{Introduction}

Candida species are opportunistic pathogens that ordinarily inhabit the human gastrointestinal tract. Colonisation of the lower respiratory tract (LRT) by Candida occurs in $25 \%$ of critically ill patients receiving mechanical ventilation and in $50 \%$ of patients suspected of ventilatorassociated pneumonia (VAP), and has been associated with longer intensive care unit (ICU) stay, a prolonged duration of mechanical ventilation, an increased risk of bacterial VAP, and possibly increased in-hospital mortality [1-4]. Whether the presence of Candida is a cause or merely a marker of a more severe clinical course is uncertain.

Furthermore, it remains unclear how to differentiate between colonisation and infection of the LRT. In general, Candida species are not assumed to be primary causative pathogens in VAP patients [5]. In a postmortem study in patients with evidence of pneumonia at autopsy, none of the subjects with a tracheal aspirate or bronchoalveolar lavage culture positive for Candida species had histopathological evidence of invasive Candida growth [6]. However, there is evidence that colonisation of the LRT by Candida species promotes the development of pneumonia by creating biofilms that are capable of holding other micro-organisms [7]. Moreover, Candida is assumed to have an indirect effect by decreasing the immune defence and favouring bacterial development [8]. Experimental and clinical studies have shown that Candida colonisation was associated with an increased risk for VAP by Pseudomonas aeruginosa, and that systemic antifungal treatment decreased the risk for $P$. aeruginosa infection in colonised patients $[1,9,10]$.

Side effects associated with systemic antifungal treatment have limited the use of pre-emptive strategies for Candida colonisation to patients who are at high-risk for invasive candidiasis, such as patients with severe and multiple-site colonisation [11]. However, local use of antifungal medication may provide a potentially attractive alternative approach. Nebulised amphotericin B (NAB) has been used to eradicate Candida species from the LRT as part of various selective decontamination of the digestive tract (SDD) protocols in patients with persistent Candida colonisation despite topical use of amphotericin B [12]. However, the clinical effectiveness of this approach in reducing the burden of Candida colonisation in ICU patients is not known.

\section{Materials and methods}

\section{Patients and measurements}

This study was performed in a 32-bed mixed ICU of the University Medical Center Utrecht in the Netherlands between April 2008 and February 2012. The Ethics Committee of the University Medical Center Utrecht approved this study and waived the need for informed consent. We analysed all mechanically ventilated adults who had been admitted to the ICU for $>72$ hours. Microbiological screening for the presence of Candida was prospectively carried out on admission and twice weekly thereafter according to a standardised protocol that was part of a SDD/Selective Oropharyngeal Decontamination (SOD) trial [12]. In brief, samples were inoculated on malt extract agar plates, and Candida load was semi-quantitatively determined (that is classified as $<10,10$ to 100 , and $>100$ colony-forming units). Colonisation was defined as the presence of Candida species in two or more consecutive bronchoalveolar lavage or sputum samples obtained on different days in the ICU, and the colonisation start date as the first positive sample. Decolonisation was defined as the absence of Candida in two consecutive samples on different days, or as the absence of Candida in the last available sample. We excluded episodes during which patients had received systemic antifungal treatment, as well as episodes during which $\mathrm{NAB}$ was initiated within the first two days after colonisation start date (before results of microbiological surveillance cultures had become available), because the reason for use of amphotericin $B$ in these cases was likely to be different.

\section{Decontamination protocol}

All patients received either SDD (from April 2008 to August 2009 and from June 2011 to February 2012) or SOD (from September 2009 to May 2011). During SDD (but not during SOD) NAB, four times $5 \mathrm{mg}$ daily, was recommended in case of Candida colonisation of the LRT [12]. To this end, $50 \mathrm{mg}$ of conventional amphotericin B for intravenous use was dissolved in $10 \mathrm{~mL}$ water for injection. For each nebulisation session $2 \mathrm{~mL}$ of water for injection was added to $1 \mathrm{~mL}$ (= $5 \mathrm{mg}$ amphotericin $\mathrm{B}$ ) of the prepared solution and aerosolised with a jet nebuliser system (Covidien, Mansfield, MA, USA). Air or oxygen under high pressure with a flow rate of 5 to $8 \mathrm{~L}$ per minute was used to generate aerosols with a mass median aerodynamic diameter of $<5$ micrometer. Each session lasted until all medication was inhaled (about 15 to 30 minutes). The nebuliser was attached to the ventilator circuit with a T-piece adaptor positioned between the endotracheal tube and the humidification filter. The nebulisation protocol did not recommend specific ventilator settings. In our clinical practice, however, patients remained on a pressure-controlled ventilator with unchanged positive end-expiratory pressure (PEEP) and inspiratory pressure settings. Depending on the mechanical ventilator type nebulisation was continuously administered during both inspiration and expiration in half of the NAB treatments, whereas in the remaining cases nebulisation was only during inspiration. Allocation of a ventilator depended on availability and was 
independent of patient characteristics. The humidification filter in the mechanical ventilation circuit was changed after each nebulisation session to avoid obstruction.

\section{Data analysis}

Because the delay in the initiation of NAB treatment (relative to the start of colonisation) may vary between patients, it is important to correct for time-varying bias, which occurs when the exposure variable is categorised to its final status rather than considering the timing of the change in status $[13,14]$. Furthermore, patients who spontaneously decolonise early have less opportunity to receive NAB. These cases contribute better outcomes to the non-exposed group and therefore may lead to an underestimation of the treatment effect. To deal with these issues, we used time-varying Cox proportional hazards regression with adjustment for age, Sequential Organ Failure Assessment (SOFA) score, Candida load at baseline, and concurrent use of corticosteroids.

In order to graphically represent the results of our time-varying analysis and correctly estimate the median time to decolonisation relative to the start of NAB [15], we used the multistate model shown in Figure 1 [16].

As a secondary study outcome, we compared the incidence rates of VAP occurring after the onset of the Candida colonisation episode in both groups. For the period 2008 to 2010, we retrospectively assessed the medical records for the occurrence of VAP, according to established CDC criteria [17]. For the period 2011 to 2012, we prospectively recorded the incidences of VAP as part of an ongoing cohort study that is aimed at finding early diagnostic and prognostic markers of sepsis [18]. Furthermore, we compared ICU mortality and remaining length of stay in ICU following the onset of Candida colonisation in patients receiving NAB compared to patients not receiving NAB.

To assess possible effect modification by the timing of treatment, we performed a secondary analysis on the overall treatment effect by comparing patients who had received NAB treatment early (within five days) versus late (after five days) following the colonisation start date. Because of a reduced number of decolonisation events in this subgroup analysis of early and late NAB starters, we tested possible confounders by adding each of them separately to a univariable Cox regression model containing only $\mathrm{NAB}$ as an explanatory variable and examining its effect on the beta coefficient of the NAB variable on decolonisation. Covariables that caused substantial confounding (that is a change in effect estimate greater than $10 \%$ ) were included in the final multivariable models. In addition, to assess possible indication bias we performed a per-protocol analysis of patients treated according to the SDD and SOD arms of the trial. Data were analysed with SAS 9.2 (SAS Institute, Inc., Cary, NC, USA) and R 2.15.1 software (package mstate).

\section{Results}

Out of a total of 1,819 patients who had been admitted to the ICU for at least 72 hours and received mechanical ventilation, colonisation with Candida occurred 401 times in 363 patients (Figure 2). Colonisation rates did not differ between the SDD and SOD periods (21\% versus $19 \%$ respectively, $P=0.44$ ). After exclusion of colonisation episodes in which concurrent systemic antifungal treatment was administered $(n=59)$ and episodes in which NAB were started within the first two days after the start of colonisation $(n=9), 333$ episodes remained for analysis.

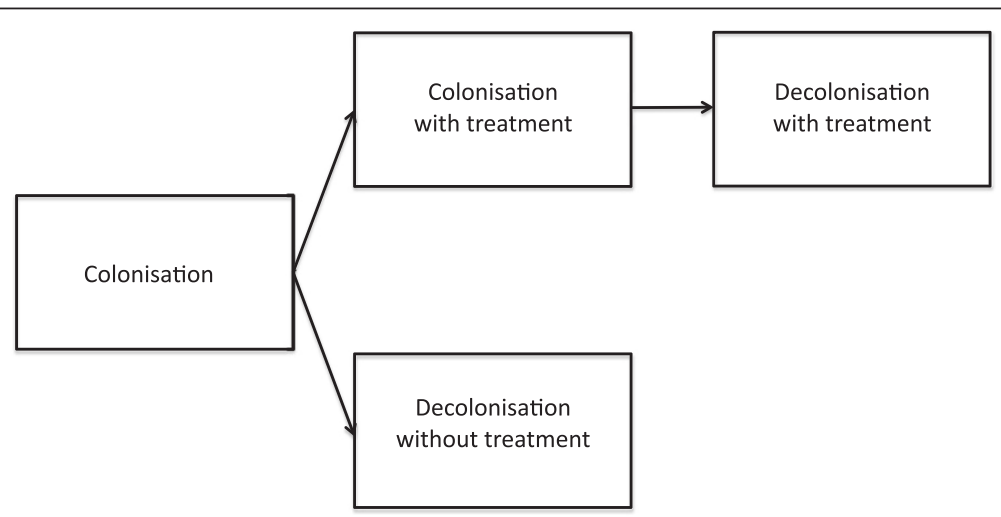

Figure 1 Multistate model. This multistate model was used for estimating the median time to decolonisation relative to the start of nebulised amphotericin B, and for graphically representing colonisation persistence probability curves in Figure 4. A patient with colonisation remained in this state as long as no treatment was given. Patients transitioned to the 'colonisation with treatment' state upon receiving treatment, and subsequently transitioned to 'decolonisation with treatment' state when decolonisation occurred. The patient underwent transition to 'decolonisation without treatment' state in case decolonisation occurred without treatment or before treatment was started. Thus, two states were transitional states, whereas the remaining two states were final states in our model (meaning that no further data were included in the model beyond this point in time). 


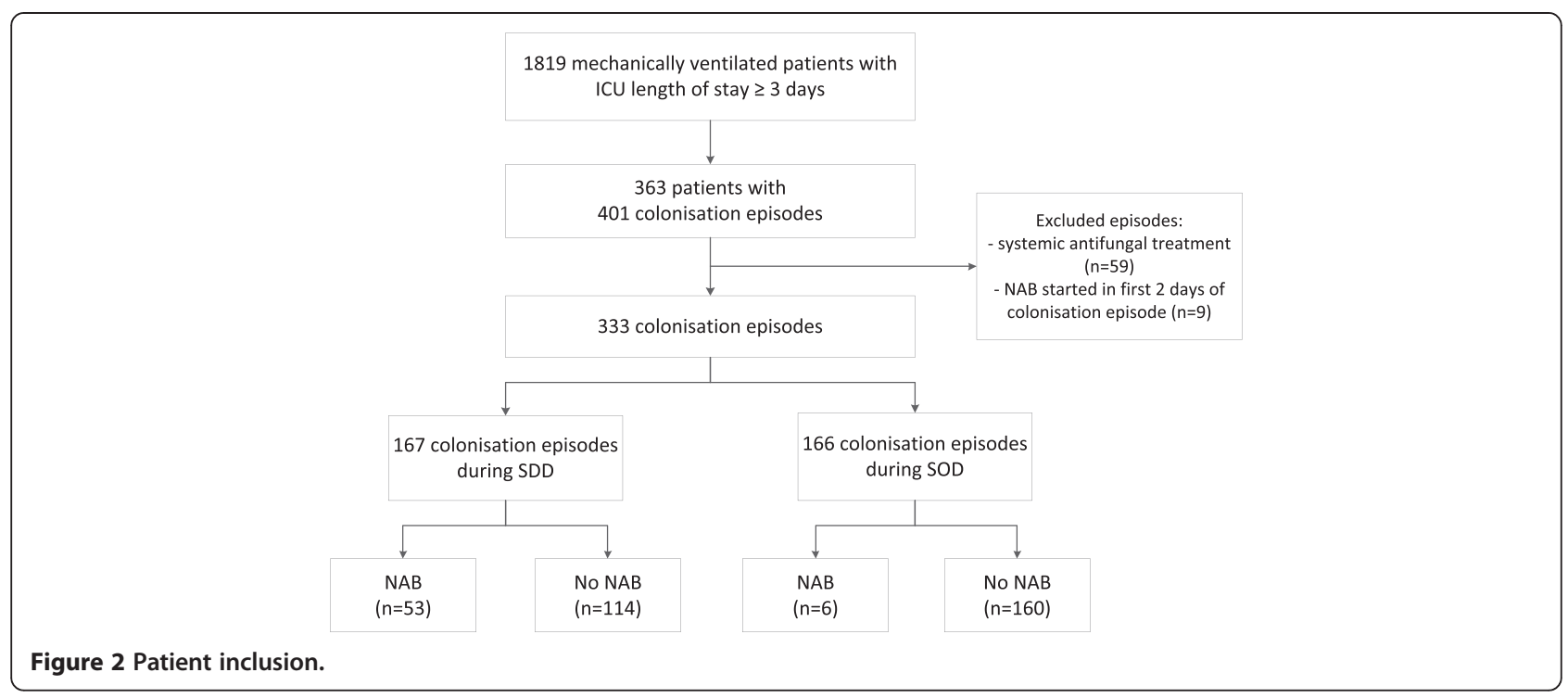

Almost half of the patients (48\%) were already colonised upon ICU admission. These patients did not have significantly more colonisation episodes compared to patients who acquired colonisation during the ICU admission $(P=0.09)$. Overall, $44 \%$ of positive cultures were classified as Candida albicans, $11 \%$ as Candida glabrata, and $8 \%$ as non-albicans/non-glabrata/non-krusei species (37\% of cultures were not further subtyped). Concurrent oropharyngeal Candida co-colonisation was present in 247 out of the 317 (78\%) initial colonisation episodes, and in 10 out of the $16(63 \%)$ recolonisation episodes.

The initial Candida load in the sputum was significantly higher in patients receiving amphotericin B compared to those not receiving therapy (Table 1). However, the number of bacterial co-colonisations was similar in both groups at baseline. There was also considerable variation in the time delay between the start of Candida colonisation and start of therapy, with $46 \%$ of patients starting treatment after more than five days (Figure 3).

Decolonisation occurred in 51 of 59 (86\%) and 170 of 274 (62\%) episodes in patients receiving and not receiving $\mathrm{NAB}$, respectively. Following the start of NAB treatment the proportion of sputum cultures growing Candida decreased over time (Table 2), suggesting true decolonisation rather than in vitro suppression of growth by amphotericin. Recolonisation occurred in $2 \%$ and $6 \%$ of patients receiving and not receiving NAB, respectively (Table 3 ).

Figure 4 shows the persistence probability of colonisation as analysed using a multistate approach. Both curves are conditioned on colonisation being present until at least day 4. Median times to elimination of Candida were estimated as six (interquartile range (IQR) 5 to 7) days and nine (IQR 6 to 20) days in patients receiving and not receiving $\mathrm{NAB}$, respectively. In time-varying analyses,
Table 1 Baseline characteristics

\begin{tabular}{|c|c|c|c|}
\hline Characteristics & $\begin{array}{c}\text { Nebulised } \\
\text { amphotericin B } \\
(n=59)\end{array}$ & $\begin{array}{c}\text { Standard care } \\
\quad(n=274)\end{array}$ & $P$ value \\
\hline Age & $63(43-76)$ & $63(52-72)$ & 0.78 \\
\hline Gender male & $43(73)$ & $189(69)$ & 0.55 \\
\hline APACHE IV score & 77 (60-96) & $75(58-92)$ & 0.42 \\
\hline Corticosteroid use $^{a}$ & $18(31)$ & $75(27)$ & 0.63 \\
\hline SOFA score ${ }^{b}$ & $6(3-8)$ & $5(3-8)$ & 0.51 \\
\hline $\begin{array}{l}\text { Days in ICU before onset } \\
\text { of the colonisation episode }\end{array}$ & $0(0-2)$ & $1(0-3)$ & 0.24 \\
\hline Candida load in sputum (CFUs) ${ }^{c}$ & & & $<0.001$ \\
\hline$<10$ & $9(15)$ & $117(43)$ & \\
\hline $10-100$ & $32(54)$ & $102(37)$ & \\
\hline$>100$ & $18(31)$ & $55(20)$ & \\
\hline $\begin{array}{l}\text { Candida colonisation of } \\
\text { the oropharynx }\end{array}$ & $50(85)$ & $213(78)$ & 0.23 \\
\hline $\begin{array}{l}\text { Candida colonisation of } \\
\text { the rectum }\end{array}$ & $23(39)$ & $75(27)$ & 0.08 \\
\hline Delay between onset of & $5(4-7)$ & NA & NA \\
\hline
\end{tabular}

Candida colonisation in

sputum and NAB start

Bacterial co-colonisation in sputum:

$\begin{array}{lccc}\text { Gram-negative rods }^{\mathrm{d}} & 13(22) & 83(30) & 0.20 \\ \text { Pseudomonas species } & 1(2) & 20(7) & 0.11 \\ \text { Gram-positive cocci } & 13(22) & 63(23) & 0.87\end{array}$

${ }^{a}$ Corticosteroid use was defined as a daily dose $>100 \mathrm{mg}$ hydrocortisone or equivalent; ${ }^{b}$ we used a modified sum score, excluding points for the central nervous system; ${ }^{c}$ Candida load at baseline was determined by semi-quantitative culture; ${ }^{d}$ Gram-negative rods including Pseudomonas species. APACHE, Acute Physiology and Chronic Health Evaluation; CFU, colony-forming units; ICU, intensive care unit; NA, not applicable; SDD, selective digestive decontamination; SOD, selective oral decontamination; SOFA, Sequential Organ Failure Assessment. Data are presented as medians (interquartile range (IQR)) or absolute numbers (\%). 


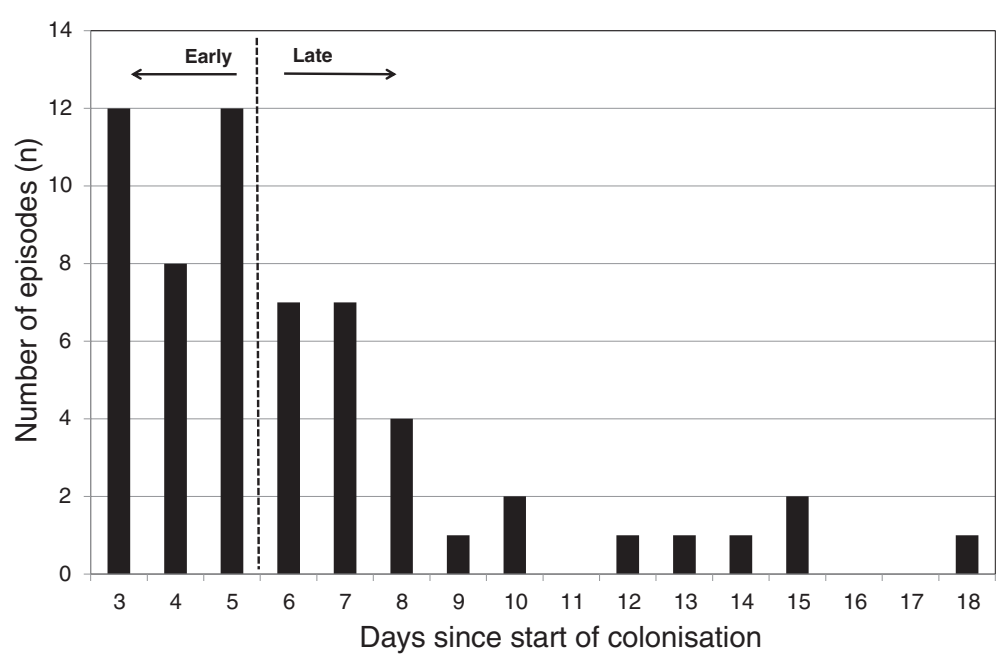

Figure 3 Time delay between start of Candida colonisation and treatment initiation. This figure depicts the observed time delay between the start of the Candida colonisation episode and the initiation of nebulised amphotericin B treatment. Episodes during which treatment was initiated within the first two days after start of colonisation (before results of microbiological surveillance cultures had become available) were excluded from the study, because the reason for antifungal treatment in these cases is likely to be different.

NAB treatment was associated with an approximately two-fold increase in the rate of decolonisation (crude hazard ratio (HR) 2.0; $95 \%$ confidence interval (CI) 1.4 to 2.7 , adjusted HR 2.2; 95\% CI 1.6 to 3.0$)$.

The incidence rate of VAP and ICU mortality did not differ between both groups (Table 3 ). The length of stay in ICU after NAB initiation was comparable to the length of stay in ICU after onset of Candida colonisation in the control group, 14 (IQR 7 to 25 ) and 14 (10 to 23) days, respectively. In 5 of 59 episodes NAB treatment was discontinued before successful decolonisation was achieved, because of extubation $(n=2)$, clinical decision to stop all antimicrobial therapy $(n=1)$ or unclear reasons $(n=2)$. No adverse effects (such as cough or bronchospasm) were documented in the medical records for any of the 59 cases.

\section{Secondary analysis}

There were 27/31 (87\%) and 24/28 (86\%) decolonisation events in early and late NAB starters, respectively, resulting in an adjusted HR of 2.0 (95\% CI 1.3 to 3.1 ), and 2.0 (95\% CI 1.3 to 3.2 ), respectively. During the SOD period, in which NAB treatment was not recommended in the protocol, NAB was applied in 6 of 166 (4\%) cases, whereas during the SDD period 114 of 167 (68\%) cases did not receive NAB while being colonised. However, after exclusion of episodes during SDD in which NAB was not started as well as episodes during SOD in which NAB was administrated (per-protocol analysis), the effect estimates remained similar (crude HR 2.1; 95\% CI 1.4 to 3.0, adjusted HR 2.2; 95\% CI 1.5 to 3.3).

\section{Discussion}

Inhalation therapy with NAB resulted in a three-day reduction in the duration of Candida colonisation in the LRT of mechanically ventilated patients who are (also) receiving topical applications of amphotericin $\mathrm{B}$ as part of a SDD protocol. This effect remained after adjustment for potential confounders and was approximately constant irrespective of the duration of colonisation prior to the start of treatment.

Although NAB has been an essential component in SDD protocols for many years [12,19], this is the first study to assess the effectiveness of inhalation treatment with NAB for eradication of Candida in colonised ICU patients. Previous studies have focused on lung transplant recipients in whom NAB was used as prophylactic treatment for, in particular, Aspergillus infection [20-22]. Inhalation treatment seems attractive, because high drug concentrations can be reached at the site of interest while reducing nephrotoxicity and drug interactions

Table 2 Proportion of consecutive sputum cultures showing Candida growth following the start of nebulised amphotericin B (NAB) treatment

\begin{tabular}{lc}
\hline $\begin{array}{l}\text { Consecutive sputum cultures } \\
\text { following the start of NAB }\end{array}$ & $\begin{array}{c}\text { Proportion of patients with } \\
\text { positive culture (\%) }\end{array}$ \\
\hline 1 & 100 \\
2 & 67 \\
3 & 25 \\
4 & 19 \\
5 & 9 \\
\hline
\end{tabular}


Table 3 Univariable analysis of secondary outcomes

\begin{tabular}{lccc}
\hline Characteristics & NAB ( $\boldsymbol{n}=\mathbf{5 9})$ & $\begin{array}{c}\text { Standard care } \\
(\boldsymbol{n}=\mathbf{2 7 4})\end{array}$ & $\boldsymbol{P}$ value \\
\hline $\begin{array}{l}\text { VAP incidence after the onset } \\
\text { of Candida colonisation } \\
\text { (per } 1000 \text { ICU days) }\end{array}$ & 6.5 & 5.5 & 0.64 \\
$\begin{array}{l}\text { Number of Candida } \\
\text { recolonisation episodes* }\end{array}$ & $1(2)$ & $15(6)$ & 0.32 \\
$\begin{array}{l}\text { Length of stay in ICU after } \\
\text { onset of Candida colonisation }\end{array}$ & $23(12-30)$ & $14(10-23)$ & 0.004 \\
$\begin{array}{l}\text { Length of stay in ICU after } \\
\text { NAB start }\end{array}$ & $14(7-25)$ & NA & NA \\
ICU Mortality & $10(17)$ & $54(20)$ & 0.62 \\
\hline
\end{tabular}

"The denominators for the calculation of the numbers of Candida recolonisation events in the NAB and standard care group are 58 and 259 initial colonisation episodes, respectively. ICU, intensive care unit; NA, not applicable; NAB, nebulised amphotericin B; VAP, ventilator-associated pneumonia. Data are presented as medians (interquartile range (IQR)) or absolute numbers (\%), except for the ventilator-associated pneumonia rate that is presented as the number per 1,000 ICU days.

$[23,24]$. However, inhalation of amphotericin B has thus far been restricted to off-label use, due to a lack of effectiveness data and standardised methods for nebulised administration. Although inhalation of NAB is generally well tolerated and considered to be safe [25], various reports of adverse effects, including cough, bronchospasm, dyspnea, nausea, and an unpleasant aftertaste, warrant prudency [26-29]. In our study, we observed only a few cases in which NAB treatment was discontinued before successful decolonisation was achieved and we did not find adverse effects documented in the medical records.

Our study has some limitations. First, the study was performed in an SDD/SOD setting exclusively. This included the daily application of a topical paste containing polymyxin $\mathrm{E}$, tobramycin and amphotericin $\mathrm{B}$ to prevent colonisation with Gram-negative bacteria and yeasts in the mouth. In addition, SDD (but not SOD) includes the use of a suspension to selectively decontaminate the digestive tract, and the systemic administration of cefotaxime during the first four days of ICU admission [12]. Oropharyngeal decontamination by oral paste might influence Candida colonisation in the lower respiratory tract over time by aspiration of topically applied amphotericin in the oropharynx. However, because in both groups the topical application was entirely the same, we assume that the estimation of the efficacy of NAB in combination with oral paste in comparison to oral paste alone is valid within the SDD setting. Both SDD and SOD have been shown to effectively reduce the incidence of VAP $[19,30]$, therefore it is possible that the clinical relevance of reducing the burden of Candida colonisation by NAB treatment will differ between settings with and without SDD.

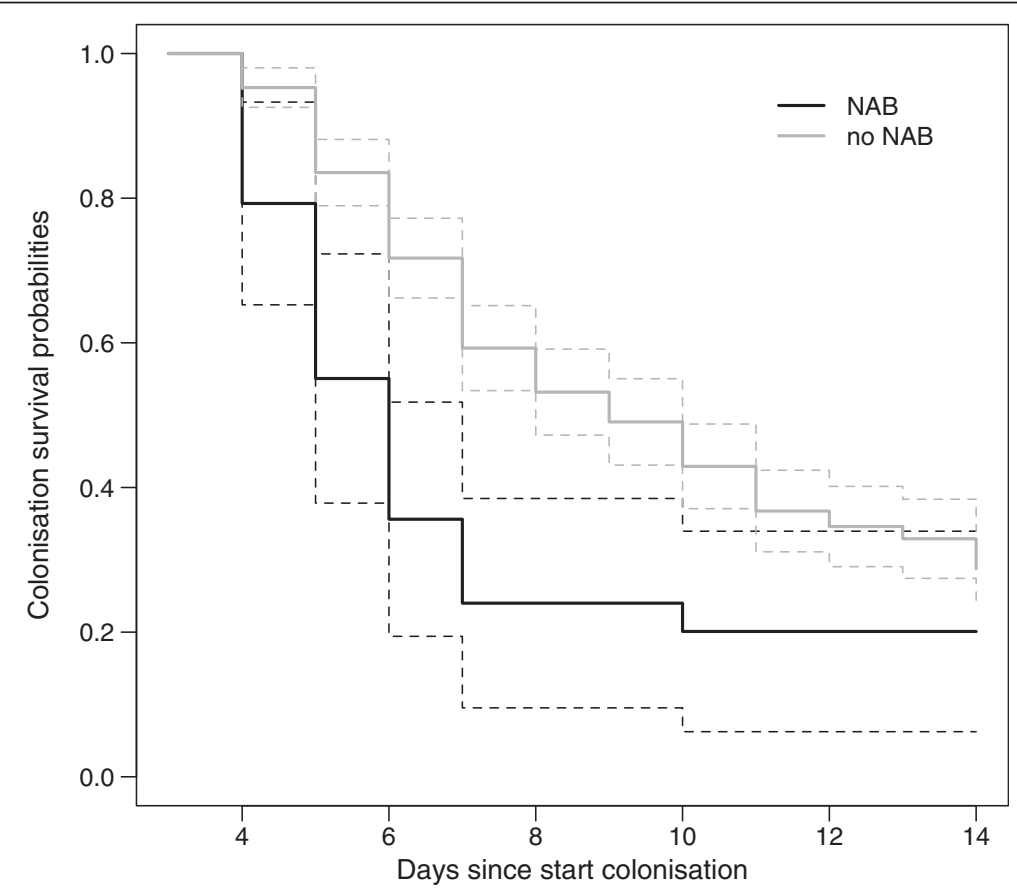

Figure 4 Time to Candida eradication of the lower respiratory tract. Colonisation persistence probability curves of the nebulised amphotericin B (NAB) group and the no NAB group were plotted with 95\% confidence intervals using the multistate approach. Both curves were conditioned on being colonised at day 4 in order to have a meaningful comparison between the treated and untreated patients. On any given day, one curve represented episodes in which NAB treatment was given and the other represented those without treatment. 
Moreover, during SDD NAB was not started in all patients eligible for this intervention according to protocol recommendations. This probably reflects indication bias. It is plausible that patients with a longer expected ICU stay or greater disease severity were more likely to receive $\mathrm{NAB}$ than patients in better health conditions. However, we reduced the possible effects of indication bias by excluding patients who received NAB before microbiological results had become available and by adjusting for potential confounding variables, such as disease severity and Candida load. Furthermore, we performed a per-protocol analysis that yielded similar results. Another source of bias may be related to the large variation in the timing of start of treatment that was observed, also known as immortal time bias [31]. Again, by incorporating a time-varying approach into our analysis, we aimed to minimise such bias.

Although we did not find a trend towards a reduced incidence rate of VAP in patients receiving NAB, we stress that the interpretation of this apparent lack of clinical effectiveness requires caution, because our study lacks the statistical power to detect potentially meaningful differences in VAP rates and because of the presence of some concurrent interventions during the use of SDD for which we did not control.

\section{Conclusions}

Inhalation therapy with $\mathrm{NAB}$ is associated with a two-fold increase in the rate of Candida decolonisation of the LRT in mechanically ventilated patients receiving SDD, resulting in an average reduction of the colonisation time by approximately three days. However, we found no evidence that effective decolonisation translates into a clinically meaningful reduction in VAP rates, ICU length of stay or mortality. The routine use of NAB in current SDD protocols, therefore, cannot be recommended.

\section{Key messages}

- Inhalation therapy with nebulised amphotericin B (NAB) is commonly used to treat colonisation of the lower respiratory tract by Candida species as part of $\mathrm{SDD} / \mathrm{SOD}$ protocols, but its effectiveness has not been studied.

- NAB therapy reduces the duration of Candida colonisation of the lower respiratory tract by approximately three days. However, the incidence rate of ventilator-associated pneumonias was not different between the two groups.

- Even without NAB treatment most patients seem to spontaneously decolonise eventually, possibly due the topical application in the oropharynx as part of SDD/SOD.
- Given the current state of evidence, NAB should not be used routinely as part of SDD.

\section{Abbreviations}

Cl: Confidence interval; HR: Hazard ratio; ICU: Intensive care unit; IQR: Interquartile range; LRT: lower respiratory tract; NAB: Nebulised amphotericin B; SDD: Selective digestive decontamination; SOD: Selective oral decontamination; VAP: Ventilator-associated pneumonia.

\section{Competing interests}

This research was performed within the framework of CTMM, the Center for Translational Molecular Medicine (http://www.ctmm.nl), project MARS (grant 041-201). Marc Bonten has received research funding from the Netherlands Organization of Scientific Research (NWO Vici 918.76.611).

\section{Authors' contributions}

$\mathrm{DO}, \mathrm{PK}, \mathrm{CS}, \mathrm{MB}$ and OC substantially contributed to the conception and design of this study. DO, PK and OC acquired the data. DO and CS performed the data analyses. $\mathrm{DO}, \mathrm{PK}, \mathrm{CS}, \mathrm{MB}$ and $\mathrm{OC}$ were involved in the interpretation of data. DO drafted the manuscript and all authors revised it critically for important intellectual content. All authors read and approved the final manuscript.

\section{Acknowledgements}

We thank Karen Vlaardingerbroek and Sandra Numan, from the Department of Intensive Care Medicine, UMC Utrecht, for their assistance in data acquisition.

\section{Author details}

${ }^{1}$ Department of Intensive Care Medicine, University Medical Center Utrecht, Heidelberglaan 100, 3584, CX Utrecht, The Netherlands. ${ }^{2}$ Department of Medical Microbiology, University Medical Center Utrecht, Heidelberglaan 100, 3584, CX Utrecht, The Netherlands. ${ }^{3}$ Julius Center for Health Sciences and Primary Care, University Medical Center Utrecht, Heidelberglaan 100, 3584, CX Utrecht, The Netherlands. ${ }^{4}$ Department of Mathematics, Utrecht University, Budapestlaan 6, 3584, CD Utrecht, The Netherlands.

Received: 9 April 2013 Accepted: 4 September 2013

Published: 11 October 2013

\section{References}

1. Azoulay E, Timsit JF, Tafflet M, de Lassence A, Darmon M, Zahar JR, Adrie C, Garrouste-Orgeas M, Cohen Y, Mourvillier B, Schlemmer B: Candida colonization of the respiratory tract and subsequent pseudomonas ventilator-associated pneumonia. Chest 2006, 129:110-117.

2. Delisle MS, Williamson DR, Perreault MM, Albert M, Jiang X, Heyland DK: The clinical significance of Candida colonization of respiratory tract secretions in critically ill patients. J Crit Care 2008, 23:11-17.

3. Hamet M, Pavon A, Dalle F, Pechinot A, Prin S, Quenot JP, Charles PE: Candida spp. airway colonization could promote antibiotic-resistant bacteria selection in patients with suspected ventilator-associated pneumonia. Intensive Care Med 2012, 38:1272-1279.

4. Olaechea PM, Palomar M, Leon-Gil C, Alvarez-Lerma F, Jorda R, Nolla-Salas J, León-Regidor MA, et al: Economic impact of Candida colonization and Candida infection in the critically ill patient. Eur J Clin Microbiol Infect Dis 2004, 23:323-330.

5. Ricard JD, Roux D: Candida pneumonia in the ICU: myth or reality? Intensive Care Med 2009, 35:1500-1502.

6. Meersseman W, Lagrou K, Spriet I, Maertens J, Verbeken E, Peetermans WE, Van Wijngaerden E: Significance of the isolation of Candida species from airway samples in critically ill patients: a prospective, autopsy study. Intensive Care Med 2009, 35:1526-1531.

7. El-Azizi MA, Starks SE, Khardori N: Interactions of Candida albicans with other Candida spp. and bacteria in the biofilms. J Appl Microbiol 2004, 96:1067-1073.

8. Ricard JD, Roux D: Candida colonization in ventilated ICU patients: no longer a bystander! Intensive Care Med 2012, 38:1243-1245.

9. Nseir S, Jozefowicz E, Cavestri B, Sendid B, Di PC, Dewavrin F, Favory R, Roussel-Delvallez M, Durocher A: Impact of antifungal treatment on Candida-Pseudomonas interaction: a preliminary retrospective casecontrol study. Intensive Care Med 2007, 33:137-142. 
10. Roux D, Gaudry S, Dreyfuss D, El-Benna J, de Prost N, Denamur E, Saumon G, Ricard JD: Candida albicans impairs macrophage function and facilitates Pseudomonas aeruginosa pneumonia in rat. Crit Care Med 2009, 37:1062-1067.

11. Lam SW, Eschenauer GA, Carver PL: Evolving role of early antifungals in the adult intensive care unit. Crit Care Med 2009, 37:1580-1593.

12. de Smet AM, Kluytmans JA, Cooper BS, Mascini EM, Benus RF, van der Werf TS, van der Hoeven JG, Pickkers P, Bogaers-Hofman D, van der Meer NJ, Bernards AT, Kuijper EJ, Joore JC, Leverstein-van Hall MA, Bindels AJ, Jansz $A R$, Wesselink RM, de Jongh BM, Dennesen PJ, van Asselt GJ, te Velde LF,

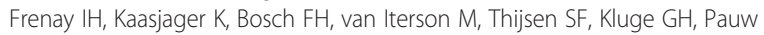
W, de Vries JW, Kaan JA, et al: Decontamination of the digestive tract and oropharynx in ICU patients. N Engl J Med 2009, 360:20-31.

13. Shintani AK, Girard TD, Eden SK, Arbogast PG, Moons KG, Ely EW: Immortal time bias in critical care research: application of time-varying Cox regression for observational cohort studies. Crit Care Med 2009, 37:2939-2945

14. Suissa S: Effectiveness of inhaled corticosteroids in chronic obstructive pulmonary disease: immortal time bias in observational studies. Am J Respir Crit Care Med 2003, 168:49-53.

15. Simon R, Makuch RW: A non-parametric graphical representation of the relationship between survival and the occurrence of an event: application to responder versus non-responder bias. Stat Med 1984, 3:35-44.

16. Putter H, Fiocco M, Geskus RB: Tutorial in biostatistics: competing risks and multi-state models. Stat Med 2007, 26:2389-2430.

17. Horan TC, Andrus M, Dudeck MA: CDC/NHSN surveillance definition of health care-associated infection and criteria for specific types of infections in the acute care setting. Am J Infect Control 2008, 36:309-332.

18. Klein Klouwenberg PM, Ong DS, Bos LD, de Beer FM, van Hooijdonk RT, Huson MA, Straat M, van Vught LA, Wieske L, Horn J, Schultz MJ, van der Poll T, Bonten MJ, Cremer OL: Interobserver agreement of centers for disease control and prevention criteria for classifying infections in critically III patients. Crit Care Med 2013, 41:2373-2378.

19. de Jonge E, Schultz MJ, Spanjaard L, Bossuyt PM, Vroom MB, Dankert J, Kesecioglu J: Effects of selective decontamination of digestive tract on mortality and acquisition of resistant bacteria in intensive care: a randomised controlled trial. Lancet 2003, 362:1011-1016.

20. Dummer JS, Lazariashvilli N, Barnes J, Ninan M, Milstone AP: A survey of anti-fungal management in lung transplantation. J Heart Lung Transplant 2004, 23:1376-1381.

21. Koo S, Kubiak DW, Issa NC, Dietzek A, Boukedes S, Camp PC, Goldberg HJ, Baden LR, Fuhlbrigge AL, Marty FM: A targeted peritransplant antifungal strategy for the prevention of invasive fungal disease after lung transplantation: a sequential cohort analysis. Transplantation 2012, 94:281-286

22. Monforte V, Ussetti P, Lopez R, Gavalda J, Bravo C, de Pablo A, Pou L, Pahissa A, Morell F, Román A: Nebulized liposomal amphotericin B prophylaxis for Aspergillus infection in lung transplantation: pharmacokinetics and safety. J Heart Lung Transplant 2009, 28:170-175.

23. Dhand $\mathrm{R}$ : The role of aerosolized antimicrobials in the treatment of ventilator-associated pneumonia. Respir Care 2007, 52:866-884

24. Dolovich MB, Dhand R: Aerosol drug delivery: developments in device design and clinical use. Lancet 2011, 377:1032-1045.

25. Kuiper L, Ruijgrok EJ: A review on the clinical use of inhaled amphotericin B. J Aerosol Med Pulm Drug Deliv 2009, 22:213-227.

26. Drew RH, Dodds AE, Benjamin DK Jr, Duane DR, Palmer SM, Perfect JR: Comparative safety of amphotericin B lipid complex and amphotericin B deoxycholate as aerosolized antifungal prophylaxis in lung-transplant recipients. Transplantation 2004, 77:232-237.

27. Dubois J, Bartter T, Gryn J, Pratter MR: The physiologic effects of inhaled amphotericin B. Chest 1995, 108:750-753.

28. Lowry CM, Marty FM, Vargas SO, Lee JT, Fiumara K, Deykin A, et al: Safety of aerosolized liposomal versus deoxycholate amphotericin $B$ formulations for prevention of invasive fungal infections following lung transplantation: a retrospective study. Transp/ Infect Dis 2007, 9:121-125.

29. Myers SE, Devine SM, Topper RL, Ondrey M, Chandler C, O'Toole K, Williams SF, Larson RA, Geller RB: A pilot study of prophylactic aerosolized amphotericin $B$ in patients at risk for prolonged neutropenia. Leuk Lymphoma 1992, 8:229-233.
30. Bergmans DC, Bonten MJ, Gaillard CA, Paling JC, van der Geest S, van Tiel $\mathrm{FH}$, Beysens AJ, de Leeuw PW, Stobberingh EE: Prevention of ventilatorassociated pneumonia by oral decontamination: a prospective, randomized, double-blind, placebo-controlled study. Am J Respir Crit Care Med 2001, 164:382-388.

31. Levesque LE, Hanley JA, Kezouh A, Suissa S: Problem of immortal time bias in cohort studies: example using statins for preventing progression of diabetes. BMJ 2010, 340:b5087.

doi:10.1186/cc13056

Cite this article as: Ong et al:: Nebulised amphotericin B to eradicate Candida colonisation from the respiratory tract in critically ill patients receiving selective digestive decontamination: a cohort study. Critical Care 2013 17:R233.

\section{Submit your next manuscript to BioMed Central and take full advantage of:}

- Convenient online submission

- Thorough peer review

- No space constraints or color figure charges

- Immediate publication on acceptance

- Inclusion in PubMed, CAS, Scopus and Google Scholar

- Research which is freely available for redistribution 